\title{
Héctor Vargas Carreño, ingeniero agrónomo y entomólogo: Un 'maestro' al servicio de la agricultura del norte de Chile
}

\author{
Héctor Vargas Carreño, agronomist and entomologist: A 'teacher' at the service of \\ agriculture in northern Chile
}

Pablo Marcos Espinoza Concha1,2*

\begin{abstract}
RESUMEN
El artículo da cuenta de parte de la historia de vida y de la visión de mundo de Héctor Elías Vargas Carreño (Navidad, Región de O”Higgins, septiembre 26 de 1941, 11 hijos), ingeniero agrónomo por la Universidad Católica de Valparaíso, magíster en Ciencias Agropecuarias por la Universidad de Chile, "Miembro Honorario" de la Sociedad Chilena de Entomología y exacadémico de la Facultad de Ciencias Agronómicas (FADECIA) de la Universidad de Tarapacá (UTA).

El viernes 24 de noviembre de 2017, en la conmemoración de los 54 años de la creación de la FADECIA, Vargas Carreño ofreció la charla "Pasado, presente y futuro de la agricultura regional". Ante una cincuentena de estudiantes y de excolegas reflexionó sobre la agricultura de los tiempos prehispánicos; el riego, la domesticación de plantas y de la agricultura nativa; el problema de la salinidad del suelo; y, como buen profesor, motivó a los futuros agrónomos a cuidar los agroecosistemas y a investigar sobre recursos fitogenéticos locales. Los dichos de Héctor Vargas Carreño dan cuenta de un profesional que, al encontrar sentido a su existencia, se realizó como agrónomo desde su particular forma de concebir sus mundos. Y, lo mejor, se puso al servicio de la comunidad de Arica, que lo reconoció como uno de los suyos "por sus méritos" en un acto público.
\end{abstract}

Palabras clave: Héctor Vargas Carreño, ingeniero agrónomo, entomólogo, visión de mundo.

\begin{abstract}
The article gives an account of part of the life history and the vision of the world of Héctor Elías Vargas Carreño (Christmas, O'Higgins Region, September 26, 1941, 11 children), agronomist from the Catholic University of Valparaíso, master's degree in Agricultural Sciences from the University of Chile, "Honorary Member" of the Chilean Society of Entomology and former academic of the Faculty of Agronomic Sciences (FADECIA) of the University of Tarapacá (UTA).

On Friday, November 24, 2017, in commemoration of the 54th anniversary of the creation of FADECIA, Vargas Carreño offered the talk "Past, present and future of regional agriculture". Before fifty students and ex-colleges, he reflected on the agriculture of pre-Hispanic times; irrigation, domestication of plants and native agriculture; the problem of soil salinity; and, as a good teacher, motivated future agronomists to take care of agroecosystems and to investigate local plant genetic resources.

Hector Vargas Carreño's sayings tell of a professional who, in finding meaning to his existence, was an agronomist from his particular way of conceiving his worlds. And, best of all, he put himself at the service of the community of Arica, who recognized him as one of their own "by his merits" in a public ceremony.
\end{abstract}

Keywords: Héctor Vargas Carreño, agricultural engineer, entomologist, vision of the world.

El viernes 24 de noviembre de 2017 fue agitado para los integrantes de la Facultad de Ciencias Agronómicas (FADECIA) de la Universidad de Tarapacá (UTA). El motivo: la celebración de los 54 años de su creación, en 1963. Bajo ese espíritu se aglutinaron los esfuerzos de autoridades, académicos, funcionarios y estudiantes para evocar, agradecer y reconocer a quienes ayudaron para que esta macrounidad desarrollara una labor fructífera en beneficio del agro del norte de Chile.
En la ceremonia oficial el decano de la época, Dr. Vitelio Goykovic Cortés, se refirió a docencia, investigación y vinculación con el medio. Luego se distinguió a la mejor egresada de 2016, Sheyla Olivares Contreras, y por años de servicio a Eugenio Sotomayor León, por 45; Patricia Pacheco Cartagena y Juan Gómez Coñajagua, por 30; Pilar Mazuela Águila y Germán Sepúlveda Chavera, por 25; Patricia Romero Romero y Héctor Vargas Ortiz, por 15; y a María Eugenia Osorio, por 10.

1 Departamento de Español, Facultad de Educación y Humanidades, Universidad de Tarapacá. Arica, Chile.

2 Programa de Doctorado en Comunicación Social, Facultad de Ciencias Sociales y de la Comunicación, Universidad del País Vasco / Euskal Herriko Unibertsitatea.

* Autor por correspondencia: pespinozac@uta.cl

Fecha de recepción: 09 octubre, 2018.

Fecha de aceptación: 10 noviembre, 2018.

DOI: 
Como eje central de la jornada, el exacadémico Héctor Elías Vargas Carreño (Navidad, Región de O’Higgins, 77 años -septiembre 26 de 1941-, 11 hijos), ingeniero agrónomo por la Universidad Católica de Valparaíso, magíster en Ciencias Agropecuarias por la Universidad de Chile y Miembro Honorario de la Sociedad Chilena de Entomología, ofreció la charla "Pasado, presente y futuro de la agricultura regional". Desde lo humano, la actividad fue emotiva, tocó fibras, removió el suelo, metáfora de conciencias. En términos prácticos, la siembra fue abundante, los futuros ingenieros agrónomos quedaron motivados para emprender sus tareas y honrar su profesión. La semilla cayó en tierra fértil. -Creo que están aburridos y me pasé en el tiempo. Es viernes, deben estar cansados. ¡Quedaría hasta aquí! -enfatizó el expositor en cuatro oportunidades. -¡No! ¡Siga! ¡Siga! ¡Siga! -fue la respuesta que se escuchó en la sala. -¡Bueno!, continúo, pero cuando quieran termino, ustedes saben -expresó.

Héctor Vargas Carreño se apoyó en vivencias, habló desde su experiencia de ingeniero agrónomo de la vieja guardia. Nada de power point o fotografías, "soy malo para esas cosas tecnológicas", dijo. Con alegría evocó sus inicios profesionales y agradeció con humildad a sus colegas. Al nombrarlos siempre antepuso "don" y luego el adjetivo de "distinguido" -otras acepciones, ilustre, noble, esclarecido- ingeniero agrónomo, profesor, científico e investigador. Se despojó sin problema de la primera persona del singular, ese prepotente 'yo', para hablar desde la primera persona del plural, ese nosotros que incluye, integra, acoge, comparte y respeta. Mismo respeto que siente y manifestó por los pueblos originarios, a los que valora y admira por la forma de vivir que tienen: en armonía con la naturaleza, no dañándola. "Los que llevan más años aquí, los alumnos mayores, me conocen, saben que soy medio repetitivo, pero es bueno remarcar las cosas", afirmó.

En líneas gruesas, Vargas Carreño comentó sobre el origen de la FADECIA y la contribución que esta ha realizado en favor de la agricultura regional en control biológico. De sus fundadores, en especial, destacó la visión del profesor Raúl Cortés Peña; y, por último, habló sobre el desafío de los ingenieros agrónomos del futuro: hacer que en los agroecosistemas se practique una agricultura sostenible, amigable con el medio ambiente.

Presentamos algunas reflexiones de Héctor Vargas Carreño expresadas ante una cincuentena de estudiantes de agronomía y de sus excolegas de la
FADECIA. Parte de su historia de vida, pinceladas de su 'pensar-actuar' que, felizmente, ejecutó en Arica durante su prolífica vida profesional. Esto queda demostrado desde la tesis que presentó para optar al título de Ingeniero Agrónomo en la Facultad de Agronomía de la Universidad Católica de Valparaíso, en 1967, Observaciones sobre la biología y enemigos naturales de la polilla del tomate, Gnorimoschema absoluta. (Meyrick). (Lep. Gelechiidae). Un resumen de esta actividad académica fue publicado en la Revista Idesia, Departamento Agricultura, Universidad del Norte, Arica (Vargas, 1970), ahora como ingeniero agrónomo, entomólogo (CICA), Departamento de Agricultura, Universidad del Norte: "Uno de los principales y tal vez más recientes problemas fitosanitarios que afectan actualmente al cultivo del tomate, en la zona comprendida entre Arica y Santiago, es la polilla Gnorimoschema absoluta (Meyrick) (Lep., Gelechiidae).

El daño causado por este microlepidóptero ha ido aumentando año tras año, lo que ha obligado a los agricultores a aplicar insecticidas en dosis crecientes y a más frecuentes y arriesgados intervalos. Esto ha provocado un incremento en los costos de producción, que por la misma naturaleza del cultivo son ya bastante elevados. Es así como se ha podido constatar en el Valle de Azapa, que es frecuente que el cultivo reciba de 10 a 15 pulverizaciones con insecticidas clorados $\mathrm{y} / \mathrm{o}$ fosforados (y a veces mezclas de ambos en 90 a 120 días). Este uso indiscriminado de pesticidas ha perturbado el equilibrio biológico, intensificando, al mismo tiempo, la incidencia del insecto sobre el cultivo.

(...) El presente trabajo fue planificado con la esperanza de contribuir a un mejor conocimiento de esta plaga, y fue desarrollado en el Laboratorio de Entomología del Centro de Investigación y Capacitación Agrícola (CICA), Universidad del Norte, Arica. Abarcó un período de 10 meses, desde enero a octubre de 1967.

Estas observaciones fundamentalmente tienen validez para la localidad en que fueron realizadas: el Valle de Azapa. No obstante, también se incluye información general que podría ser de utilidad en otras zonas, donde la polilla representa un problema para el cultivo del tomate", sostuvo.

Y, como ha sido su costumbre, Héctor Vargas Carreño agradeció: "Al profesor Raúl Cortés P., por haber revisado y corregido pacientemente el texto, y por sus útiles consejos. Al Dr. Leopoldo E. Caltagirone, a quien pertenece el esquema general 
adoptado en la presentación de este trabajo. Al Ing. Agr. Sr. Sergio Rojas, por sus valiosas sugerencias, y por las facilidades de laboratorio y biblioteca, ofrecidas en la Subestación Experimental La Cruz.

Agradezco también la ayuda y el estímulo recibidos de mis compañeros y colegas: Alfonso Aguilera P., Mauricio Jiménez R., Javier Acuña G., Juan Pacheco W. y Enrique Zúñiga S. Y, finalmente, agradezco a todas las personas que, de uno u otro modo, hicieron posible esta investigación", manifestó. Sin duda, ellos también merecen un homenaje.

Ese viernes 24 de noviembre de 2017 sus dichos sonaron potentes en el Valle de Azapa, el mismo que con su trabajo tesonero, constante e inquieto ayudó a desarrollar para bien de las economías familiares y locales.

A más de un año de esa clase magistral, plasmamos estas líneas como testimonio de gratitud a un ingeniero agrónomo que eligió a Arica para desarrollarse como hombre de bien y como profesional íntegro. Botón de muestra es la retroalimentación a la noticia aparecida en el portal de la Universidad de Tarapacá. Exacadémico y fundador de la actual Facultad de Ciencias Agronómicas fue distinguido por la Sociedad Chilena de Entomología. La bajada afirmó: "Héctor Vargas Carreño ha contribuido con su trabajo históricamente al conocimiento de los insectos en Chile. Publicado por Facultad de Ciencias Agronómicas (FADECIA). 19 DE MARZO, 2018. 792 visitas". Gran parte de ellas escribieron felicitaciones y mensajes de gratitud al hoy exprofesor. (Ver www.uta.cl).

Misma actitud que hace cincuenta años destacó Alfonso Aguilera Puente (1968), ingeniero agrónomo, entomólogo del Centro de Investigación y Capacitación Agrícola, en Separata de la Revista de la Universidad del Norte, La Colección de Insectos del Centro de Investigación y Capacitación Agrícola, (CICA), ARICA. "También agradecemos en forma especial a los principales colaboradores y funcionarios del CICA, señores Héctor Vargas Carreño, Ingeniero Agrónomo; Mauricio Jiménez Roco, Técnico Agrario y Gerardo Díaz Pizarro, Ayudante de Laboratorio, quienes con verdadera dedicación han contribuido a formar la colección de nuestro Centro".

Para nosotros, que nos oponemos al olvido, un retazo de memoria histórica de un "excepcionalnormal", un hombre que dentro de su 'normalidad' de ingeniero agrónomo 'observó algo excepcional' que ayudó a la agricultura local desde su especificidad, la entomología. Esfuerzos reconocidos por la comunidad de Arica, a través de la Ilustre Municipalidad, el sábado 8 de septiembre de 2018 al distinguirlo "PorSus Méritos". Por lo anterior, con este homenaje pretendemos que la visión de mundo de Héctor Elías Vargas Carreño se materialice en un 'no al olvido' y que sus reflexiones no se las lleve el viento. Por último, consideramos que “don" Héctor es uno de tantos hombres y mujeres silentes que contribuyeron a desarrollar el entorno social donde vivieron y convivieron y, por lo tanto, es 'portador de conocimiento relevante'. Con ello, el paso del tiempo 'no puede ni debe' negarlo a las futuras generaciones.

\section{Mirar al pasado}

Esas palmas de abanico las pusimos nosotros, dan cuenta de los cincuenta y tantos años que tiene esta unidad. Si hay alguien que tuvo el verdadero mérito de ver la necesidad de crear una institución agrícola en esta zona fue don Raúl Cortés Peña. Él había sido profesor en la Universidad de Chile, donde estudió, y en la Pontificia Universidad Católica de Santiago. Conocía muy bien los problemas del norte, porque trabajó como funcionario del Ministerio de Agricultura, dondellegó a los cargos más altos: director del antiguo Departamento de Defensa Agrícola, que precedió al Servicio Agrícola y Ganadero (SAG), y del Departamento de Investigaciones Agrícolas, que antecedió al Instituto de Investigaciones Agropecuarias (INIA). Tenía una visión clarísima de la realidad agrícola del país y, particularmente, de esta zona. En los años de 1940 demostró un problema enorme de desequilibrio: habían llegado plagas nuevas, por ejemplo, la conchuela negra del olivo (Saissetia oleae), que arruinó la producción olivícola de esa época, y la conchuela hemisférica (Saissetia coffeae). Ambas poseen control biológico, pero acá no lo tenían.

Don Raúl trabajó en algunos proyectos de esa naturaleza, y sabía muy bien cuáles eran las dificultades, porque mantenía contacto con los agricultores. Ese es otro aspecto a destacar: el profesional ve y aprecia mejor un problema agronómico a través del vínculo diario con el agricultor. Él vino y se quedó acá, anduvo por el valle y tomó contacto con quienes trabajaban aquí, por ejemplo, con don Hugo Trivelli, quien llegó a ser ministro de Agricultura. Don Raúl manejaba información y, además, los agricultores le contaban sus dificultades. Luego propuso la creación de un laboratorio, hubo una priorización de problemas y las plagas eran lo más urgente por resolver. 
Otro tema: el agua, muy poca, todavía no llegaba el canal Lauca, que inició su funcionamiento en 19621963. Cuando empezamos a trabajar acá estaban canalizando el valle, la idea era hacer llegar agua a la parte baja. Entonces, dijeron, las plagas son lo primero, con poca agua no se puede seguir produciendo, y crearon el laboratorio. Mi tarea fue hacer un catastro de los insectos nocivos, las plagas, y también de los benéficos.

\section{El problema de la salinidad del suelo}

Una de las cosas que más llamaron mi atención, no es de mi especialidad y la veo como amenaza grave para la agricultura de la región, fue el problema de la salinidad. Recuerdo que visité a un agricultor que tenía inconvenientes con el olivo. Utilizaba 'pindongos', esos barriles de roble para las aceitunas, los de ahora son de plástico. Le pregunté de dónde traía la sal. "¡Es fácil! Mire, allá, en el borde del cerro, de ahí saco la sal", me dijo. Para mí fue como un campanazo. Tenía la idea de que el desierto estaba cubierto de arena, por lo leído y lo visto en películas, pero no un conocimiento real del ambiente en el que trabajaría.

Tenemos que remontarnos a millones de millones de años atrás, es importantísimo saber los orígenes de los problemas. Atacama, de acuerdo a publicaciones de 2006 o de 2008, hay consenso en que es el desierto más antiguo del mundo, que se formó después de la separación de la Pangea, un supercontinente en los periodos Paleozoico y Mesozoico. Eso quedó claro hace más de un siglo cuando Alfred Wegener, un científico alemán, climatólogo, comparó mapas y vio que había coincidencias entre África y América del Sur y también con el hemisferio norte. Investigaciones sobre placas, complementarias con la anterior, demostraron que América se separó y continúa separándose, ahora a un ritmo muy bajo. En ese proceso se fragmentaron las montañas y los cerros, el "Sombrero" y otros quedan como vestigios del volcanismo. La tierra tiene como 4.600 millones de años y la separación como 300 millones de años. El desierto de Atacama empezó a formarse ahí.

Quedó una zona donde el Anticiclón del Pacífico estabiliza el tiempo, no hay condiciones para que se generen muchas lluvias. Después se agravó este problema, a medida que por este choque de placas se fue levantando la cordillera de Los Andes, que evita que se forme una capa de inmersión térmica que hace más estable el tiempo atmosférico. De esa forma no llueve nunca. Científicos reconocen que este es el desierto más árido y el más antiguo del mundo. ¿Qué significa? Que es mucho más grave lo que me dijo aquel agricultor. Por millones de años, lo que vemos fue fondo marino y, al elevarse y no haber lluvias, se transformó en el desierto de Atacama, ¡de ahí la sal! Esto es, a mi juicio, uno de los problemas más serios que se le plantean a la agricultura del futuro. Habría que ser brujo para hablar con autoridad de lo que pasará, pero sí podemos adentrarnos en cuáles han sido las dificultades.

Según los científicos, la última gran glaciación duró casi 100.000 años: empezó hace 110.000 años y terminó hace 12.000 años. En ese período se formaron grandes glaciares sobre la tierra, el nivel del mar comenzó a bajar, lo contrario a lo que pasa ahora con el calentamiento global. La estimación es que bajó entre 100 y 120 metros, todo congelado. Este continente, separado del resto y que había tenido una evolución, pudo albergar las migraciones que arrancaban de las hambrunas y pasaron en distintas oleadas por el puente de Bering. Ellos son los fundadores reales y los que domesticaron los primeros cultivos en América. No podemos dejar de agradecerles.

Lo que hacen en el Museo de San Miguel de Azapa -mediante la arqueología y la antropología- es demostrar que aquí se establecieron tempranamente grupos de pescadores, recolectores y cazadores. Hace más de 10.000 años encontraron un ambiente propicio para vivir, agua dulce, caza, pesca y algunas plantas que pudieron recolectar, por ejemplo, el fruto del chañar o el tomatillo, para completar su alimentación. Así llegaron los chinchorro, tanto aquí como en Camarones, y principalmente acá porque había agua dulce.

\section{Riego, domesticación de plantas y agricultura nativa}

Las cuencas de Azapa y Vítor son las únicas que contienen agua dulce y desembocan al Pacífico. Arriba (altiplano) hay otras, pero van hacia el este, al Atlántico; son endorreicas, el agua no tiene salida fluvial al océano. Es una ventaja enorme que tiene el Valle de Azapa, que hoy está en crisis. ¿Qué hicieron los habitantes que se establecieron acá? Tomaron plantas que crecían alrededor de sus refugios. Una de las hipótesis más aceptadas sobre el origen de la agricultura es la del "montón de basura o de desechos", y la propuso un botánico inglés hace unos 25 o 30 años. En todos los grupos, como 
los chinchorro, en la basura dejada crecen plantas, unas con mayor facilidad, las nitrófilas (habitan en suelos con alto contenido en nutrientes como nitratos, potasio, fósforo). Daban mejor y se podían defender de los animales que llegaban a comer. Así empezaron a domesticarlas, por ejemplo, el tomate. Se dice que los primeros procesos fueron en México, pero eso sucedió en todo el mundo.

Las migraciones llegaron hasta Tierra del Fuego. Estudios genéticos demuestran que los huilliches son muy cercanos a los changos del norte y estos a los chinchorro. En todo Chile se domesticaron plantas, y en esta zona, por lo menos 70 especies fueron usadas por la agricultura precolombina. Las más conocidas son la papa, la quínoa y el maíz.

La conquista de América fue peor que un tsunami o un megaterremoto para la agricultura nativa. ¿Por qué? Porque los conquistadores venían, la mayoría de ellos, con el deseo de hacer fortuna rápido y regresar a España para comprar títulos nobiliarios. Era un fin que a cualquiera seduciría, pero implicó que tuvieran un trato inhumano con las poblaciones nativas y con todo el que se les oponía. Entre ellos pelearon. Está el caso de los Pizarro: Francisco mató a su hermano que vivía en el Cusco, porque este reclamaba mayor libertad, tuvo apego por los indígenas.

¡Aquí se esclavizó! ¡Hay que repetirlo una y otra vez! Cuando estudié historia se decía que Chile había sido el único país donde no había existido esclavitud, pero es una mentira del porte de un buque. Ahora lo sabemos por las investigaciones y por los manuscritos de la época, que fueron ocultados, y los que los conocían guardaron silencio.

Un jesuita publicó un libro, en 1670, para denunciar todos los atropellos de La Frontera, en el sur. Si ahora tenemos el problema en La Araucanía es, en gran medida, por eso. Se dice que la guerra duró trescientos años, pero eso no explica el problema. Lo cierto es que se instaló un negocio de esclavitud para enviarla al centro del país y a las minas del Norte Chico y, sobre todo, al Perú, que tenía una mayor capacidad económica. Se cometieron barbaridades, llevaron niños y niñas desde ocho años hacia arriba, mujeres y hombres jóvenes, a los viejos los descartaron. No se hizo caso de ello. Un investigador chileno, profesor de la Universidad de Colorado, Estados Unidos, publicó la denuncia del jesuita Diego de Rosales, quien vivió en La Araucanía y tuvo el valor de exponer estos atropellos. Había corrupción, o sea, en Chile ha existido desde la colonia y cuesta erradicarla, está metida en nuestro ADN.
Junto con esclavizar, el factor principal para la agricultura es que se hizo lo mismo con los que conocían los cultivos nativos, que fueron demonizados. La quínoa, por ejemplo, no se debía comer, porque a los originarios no se les consideró, se dudó de que tuvieran alma. Felizmente, hubo gente que los defendió, como Diego de Rosales, pero la mayoría calló. Ese libro es fundamental, debiera estar en la biblioteca.

Una de las cosas que cuesta para hacer cambios en la agricultura de zonas áridas es que al nativo se le debe considerar, primero, por quien le lleva el mensaje. Muchas veces ellos le temen al visitante. ¡Esto de demonizar los cultivos andinos! Los colonizadores se dieron cuenta de que para explotar las minas tenían que alimentar a los esclavos y la papa fue lo más barato. El chuño, descubierto miles de años antes, les sirvió para los viajes a vela que tenían que hacer a España. Así valoraron estas especies y la papa fue llevada a Europa.

Los humanos tenemos que sufrir para darnos cuenta de las cosas, y a veces tienen que pasar generaciones. Por ejemplo, la papa llegó a ser el principal cultivo en Irlanda, los ingleses no la comían porque son 'distintos'. Irlandeses, holandeses, alemanes, franceses y los españoles, en menor medida, consumieron tantas que, accidentalmente, se llevó desde acá el tizón de la papa (Phytophthora infestans), que no tenían. Trabajaban materiales libres de este, y perdió la resistencia. Aunque se multiplica vegetativamente, debe haber habido algunas variedades que se formaron por reproducción sexual, por la baya que da. Y vino la hambruna de Irlanda. Un tercio de esa población murió de hambre y el otro tercio emigró a Estados Unidos, mayoritariamente. El país quedó reducido a un tercio.

Lo importante es que hay un gran número de cultivos andinos y es el desafío para los jóvenes. ¿Cuántos son los cultivos andinos que se desarrollan hoy en Chile? Pocos. La papa, porque está en esta zona y en Chiloé. Hay un principio ecológico que es importante recordar y que aprendí de don Leopoldo Caltagirone, un distinguido ingeniero agrónomo que se doctoró en la Universidad de California, y se transformó en profesor allá. Por iniciativa de don Raúl Cortés vino a Chile, nos hizo la asignatura de Biología General y Genética, tuvimos la suerte de aprender de él. Nos enseñó que en la distribución hay una correlación entre la altitud y la latitud, por eso se explica por qué en Chiloé hubo un centro de desarrollo de papas: la domesticaron los huilliche y los mapuche de más al sur. Allá existe papa silvestre, por esa correlación entre altitud y latitud. 
Esto lo comprobamos después con don Raúl Cortés en entomología. Él había descrito unos taquínidos de la zona central que se trató de establecer en Arica, pero no se pudo. Al poco tiempo, arriba, encontramos que había Incamyia chilensis. Aquí funciona sobre 2.000 metros y a nivel del mar en la zona centro sur.

La papa y el maíz por sí solos ganaron espacio, y están entre los cuatro o cinco recursos más importantes del mundo entero. La papa, sobre todo. Al maíz en algunas partes se le mira con recelo, porque es alimento para animales, pero no se dan cuenta de que hay una variedad botánica, como la que se utiliza para hacer harina, que tiene un valor mucho mayor que el usado para alimentación animal.

Hay ene especies que están esperando que gente joven las estudie. Por ejemplo, aparte de la quínoa, existe otra especie del mismo género, la cañihua (Chenopodium pallidicaule), mucho más tolerante al frío y se puede cultivar sobre los 3.500 a 4.000 metros, ideal para la zona alta de Arica. En Perú y en Bolivia existe, es tanto o más nutritiva que la quínoa, y en Chile nadie lo ha hecho.

Otra cosa: los precolombinos y los agricultores actuales, que son el resultado del mestizaje, no solamente cultivan, sino que también recogen, colectan. En la zona sur, la frutilla (Fragaria chiloensis) o las setas que crecen en el suelo y en los troncos de los árboles se pueden cultivar, tienen gran valor nutricional. También el maqui (Aristotelia chilensis), esta baya que ha despertado interés, y la murta (Ugni molinae), entre otras. Así se defendieron los mapuche en esta pseudoguerra de Arauco, recolectando material, porque los correteaban para esclavizarlos. Aquí, todavía hay cosas que se colectan en la precordillera. Cuando trabajábamos en transferencia de tecnología nos dimos cuenta de que en marzo la gente iba a recolectar los frutos del cereus, que es muy agradable, y hacían bebidas refrescantes. Es una cactácea (Browningia candelaris).

\section{Mirada de futuro}

Lo hecho, que puede ser considerado importante, es mínimo con todo lo que se debe realizar para desarrollar completamente esta región. Estamos en un lecho marino, sal por todos lados, no llueve nunca y se agregan los aerosoles marinos. Se producen en todo el mundo por efecto del oleaje, el viento en la superficie del mar. Donde llueve se anulan, y esas sales van a dar al mar por el curso de los ríos. En Arica no ocurre eso, los aerosoles marinos vienen hacia el continente y caen sobre la franja costera. Con vientos fuertes llegan muy adentro, se mezclan con el polvo que, luego, es llevado, y salinizan las superficies. Por eso es que en este desierto las arenas no son livianas, no quedan huellas, y salvo algunas dunas, el suelo es duro como el cemento.

Lo que digo es el punto neurálgico a resolver si queremos que esto siga siendo productivo. Hay índices alarmantes, no hay conciencia de que estamos en una cuenca. Cuando en 1962 se conectó el canal Lauca comenzó a traerse a ella (la cuenca) algunos elementos que no existían: el boro. ¿Quién estudia el boro?, ¿cuánto ha aumentado aquí? Se sabe a través del Laboratorio de Química de nuestra facultad que los análisis reflejan lo que viene por la nutrición de las plantas. Se aplican grandes cantidades de nitrógeno. El agua de acá contiene niveles altos de nitratos, ¡no sé cómo lo hace la planta de agua potable para bajar esos índices! ¡Es cosa de mirar el sarro que se forma en los hervidores de agua!

Sobre agricultura precolombina se hizo un bonito estudio. Un colega, especialista en suelo, nuestro amigo Alfonso Osorio Ulloa, un distinguido ingeniero agrónomo, investigó 'las bases intercuencas precolombinas'. Las poblaciones indígenas tenían plena conciencia del problema de la salinidad. En Lluta había más agua, llevaron el Vilasamanani a Socoroma por una obra de ingeniería que hicieron a 'ñeque' no más, no conocían la rueda y no había animales de fuerza, como en Europa. Establecieron un sistema de conexión al Vilasamanani, la fuente del vivir del San José, y habitaron Socoroma.

Además, ellos tenían otra práctica que se haperdido. Lo he dicho una y otra vez, pero lo repito porque refleja la sabiduría de los pueblos precolombinos. En algunas partes se mantiene, pero aquí se ha perdido, porque se fueron poblando el altiplano y la precordillera: desalinizar los bofedales. Cuando llegaron los conquistadores mucha población huyó hacia las alturas y vivieron de esos cultivos resistentes a las bajas temperaturas. También de la ganadería, criaban llamas y alpacas. ¿Cómo mantenían el sistema? Esto contado por gente que conocí hace décadas: todos los años antes del comienzo de las lluvias, desalinizaban los bordes de los bofedales o humedales de altura, que están en hondonadas y tienen un drenaje superficial pobre, por eso existen.

Son un reservorio de agua importante y muy valioso de hierbas tiernas útiles para las alpacas, que sólo comen en ellos. Hoy día no sé si en el bofedal 
de Caquena lo hacen, los otros fueron abandonados. Con el olvido de esa práctica se han ido salinizando y eso causa un enorme daño al sistema hidrológico, porque decae y son incapaces de retener el agua. Los bofedales no sólo cumplen la misión de alimentar a las alpacas y a las especies silvestres, como la vicuña, sino que también reemplazan a los bosques de la zona sur. Acá no hay bosques, salvo la queñua (Polylepis spp.), que es muy rara, pero no desempeña el rol de retención de agua.

Lo que falta en Arica es establecer un sistema para definir el flujo ecológico de una cuenca. Cuando llegué, la ciudad estaba llena de este sistema tradicional de riego: los caracoles. Se inventaron porque es la forma natural de aprovechar bien el agua, que lentamente da vueltas y vueltas. Con eso se conseguía desalinizar el perfil del suelo. Por una parte, que quedaba por las vertientes y las avenidas, si el agua había sido buena arriba (altiplano), entonces, desalinizaban el perfil y contribuían a mejorar cuando venían las avenidas, porque llegaba material fino. Eran suelos esencialmente arenosos. En el Valle de Azapa, los de mejor calidad se cultivaron con el sistema de caracol.

\section{¿Cómo mejorar los suelos?}

Con algo que la Organización de las Naciones Unidas para la Alimentación y la Agricultura (FAO) ha venido predicando desde hace mucho tiempo: la agricultura sostenible o sustentable. Distinto a alterar el perfil del suelo. Lo que se hace con la maquinaria es pensar en la cosecha próxima y no a largo plazo, es una tremenda perturbación para el suelo darlo vuelta todos los años. De este cambio se habla desde hace mucho tiempo, desde cuando estudié agronomía, pero nadie consideraba la 'mínima labranza' o la 'labranza cero'.

En una zona donde el agua es escasa y la materia orgánica se descompone muy rápido por el calor, esto ya lo hacían los pueblos originarios con el sistema del caracol. Se construía una vez y, después, realizaban una labranza mínima.

Lo que hace falta, y esta facultad podría ayudar, es determinar el caudal ecológico que se requiere en las distintas secciones de la cuenca hidrográfica y de la zona altiplánica. Estamos unidos a la cuenca del río Lauca, por lo tanto, se debería trabajar en ese tema que, entiendo, jamás se ha determinado. La definición de caudal ecológico dice: Es un instrumento de gestión que establece la cualidad, la cantidad y régimen del flujo de agua requerido para mantener los componentes, funciones, procesos y la resiliencia de los ecosistemas acuáticos que proporcionan bienes y servicios a la sociedad.

A modo de ejemplo preocupante, todos quieren más superficie y han invadido el cauce del río. Para hacerlo arrasaron con la vegetación ribereña. Cuando este valle se comenzó a regar con aguas del Lauca eran 1.200 a 1.500 hectáreas; después, con la Reforma Agraria se agravó. Es bueno que crezca el área de cultivo, pero tiene que ser con prudencia. La vegetación ribereña es esencial para mantener el equilibrio, porque hay muchas especies asociadas a ella. Uno de los peores problemas fitosanitarios que tiene el olivo hoy es la conchuela móvil del olivo (Orthezia olivícola), que vivía en la vegetación ribereña, en las chilcas (Baccharis latifolia), en el molle (Schinus molle). Entonces, hay que asegurar que todos los componentes originales y los introducidos puedan salvaguardarse, de manera tal que los frutos que da la agricultura sean los mejores.

En la definición de caudal ecológico no captamos bien la palabra resiliencia: la capacidad de tolerar las perturbaciones que pueden ser de carácter natural, una avenida, una sequía o el fenómeno de El Niño. También las prácticas agrícolas, como las entrópicas, porque manejamos mal el sistema. Ese es el desafío de ustedes, los agrónomos jóvenes. ¡Tener una visión ecológica de la agricultura!

Han venido expertos para ver lo de la salinidad. La primera vez que lo escuché en forma clara fue al Dr. Goldman, de Israel. Hay un informe que escribió junto a don Juan Pacheco (Q. E. P. D.), nuestro colega que fue director del CICA, sobre lo que se proponía para esta institución. Se contrataría a una persona para que estudiara la salinidad, los balances hídrico y salino de la cuenca. ¡Jamás lo hemos hecho! Estamos en deuda, porque es la amenaza más seria que tiene esta cuenca: salinizarse y convertirse en desierto.

Existen antecedentes históricos buenos de recordar: Mesopotamia, un granero para los países de Asia Menor, por el mal uso del agua salinizaron el suelo y decayó. Con esa lección aprendida, dolorosamente, resurgieron. Eso lo tenían muy claro los pueblos antiguos, pero nosotros somos el fruto de una migración intercontinental. Con sabiduría comenzamos a construir una agricultura floreciente y, luego, vino la hecatombe con la conquista. Tenemos que recuperar ese conocimiento ancestral y usarlo bien, para que las futuras generaciones puedan disfrutar las bondades de Arica. 


\section{Literatura citada}

Vargas, $\mathrm{H}$.

1970. Observaciones sobre la biología y enemigos naturales de la polilla del tomate, Gnorimoschema absoluta. (Meyrick). (Lep. Gelechiidae). Revista Idesia, 1: 75-110.
Aguilera, A.

1968. La Colección de Insectos del Centro de Investigación y Capacitación Agrícola (CICA), ARICA. Revista de la Universidad del Norte, II (1): 3-14. 